TITLE:

\title{
Ab initio study of magnetism at iron surfaces under epitaxial in-plane strain
}

\section{$\operatorname{AUTHOR}(\mathrm{S})$ :}

Shimada, Takahiro; Ishii, Yoshiyuki; Kitamura, Takayuki

\section{CITATION:}

Shimada, Takahiro ... [et al]. Ab initio study of magnetism at iron

surfaces under epitaxial in-plane strain. Physical Review B 2010, 81(13): 134420.

\section{ISSUE DATE:}

2010-04

URL:

http://hdl.handle.net/2433/129640

RIGHT:

(C) 2010 The American Physical Society 
PHYSICAL REVIEW B 81, 134420 (2010)

\title{
$A b$ initio study of magnetism at iron surfaces under epitaxial in-plane strain
}

\author{
Takahiro Shimada, ${ }^{*}$ Yoshiyuki Ishii, and Takayuki Kitamura \\ Department of Mechanical Engineering and Science, Kyoto University, Sakyo-ku, Kyoto 606-8501, Japan \\ (Received 9 February 2010; revised manuscript received 29 March 2010; published 16 April 2010)
}

\begin{abstract}
We investigated magnetism at the (001) surface of iron and its response to the epitaxial in-plane strain that corresponds to bcc-fcc (Bain's) transformation path using ab initio (first-principles) spin-density-functional theory calculations within the generalized gradient approximation. The magnetic moment is enhanced at the surface of a ferromagnetic (FM) film under a strain-free condition. This was caused by electron rearrangement from the minority- $t_{2 g}$ to majority- $t_{2 g}$ state due to the decrease in nearest neighbors at the surface. Under in-plane strain, the magnetic and structural phase transition from the FM-bcc to double-layerantiferromagnetic-fcc occurred at the critical strain of $\varepsilon=-0.09$, accompanying directional bond switching from the nearest-to second-nearest neighbors in the minority spin. The transition caused a discontinuous change in the magnetic moments on the inner layers of the film across the transition, while the magnetic moment of the surface layer was rather insensitive. This was because the electron rearrangement from the $t_{2 g}$ to $e_{g}$ states during the transition was limited to within the minority spin due to the fully occupied majority spin state.
\end{abstract}

DOI: $10.1103 /$ PhysRevB.81.134420

PACS number(s): 75.70.-i, 73.22.-f, 64.70.Nd, 31.15.A-

\section{INTRODUCTION}

In recent years, nanostructured materials have attracted considerable attention and research because of their unique magnetic properties with respect to those of the bulk ${ }^{1-3}$ and their potential technological applications, e.g., high-density magnetic memory and sensors. ${ }^{4-7}$ Transition-metal magnetic ultrathin films and multilayers are, in particular, of central interest due to their complex magnetic phase diagram. For example, face-centered-cubic (fcc) $\gamma$-Fe ultrathin films epitaxially grown on $\mathrm{Cu}(100)$ substrates exhibit a variety of magnetic properties, ${ }^{8}$ while body-centered-cubic (bcc) $\alpha$-Fe exhibits a ferromagnetic (FM) ground state. Theoretical studies based on $a b$ initio (first-principles) spin-densityfunctional theory (DFT) calculations have mostly contributed to this issue and have revealed that the magnetic ground state of $\gamma$-Fe ultrathin films depends sensitively on their thickness and symmetry. ${ }^{9-12}$

Iron thin films grown epitaxially on a specific substrate are subject to in-plane tension or compression due to lattice mismatch at the interface between the film and the substrate. The resulting in-plane strain induces tetragonal distortions to the lattice of film, which lead to a crystalline structure transformation from bcc $(\alpha)$ to fcc $(\gamma)$. The deformation from bcc to fcc is known as the (epitaxial) Bain's transformation path ${ }^{13}$ and has been used to theoretically investigate the magnetic phase diagram of transition-metal thin films grown on various substrates: ${ }^{14-19}$ Friák et al. ${ }^{14}$ investigated the magnetic phase boundaries in iron along the Bain's path with various volumes using $a b$ initio full-potential linearized augmented plane-wave (FLAPW) method, which included the collinear magnetic orderings of the FM, nonmagnetic (NM), single-layer-antiferromagnetic $\quad(\mathrm{AFM} 1-\uparrow \downarrow \uparrow \downarrow \ldots)$ and double-layer-antiferromagnetic (AFMD- $\uparrow \uparrow \downarrow \downarrow \ldots)^{9}$ states. They showed that theoretically predicted FM/AFMD/AFM1 phase boundaries agreed well with the experimental observations within the generalized gradient approximation (GGA). ${ }^{20,21}$ In recent years, Tsetseris ${ }^{15}$ and Okatov et al. ${ }^{18}$ further included the noncollinear spin-spiral (SS) state in the magnetic phase diagram of iron. In these studies, however, there was little discussion of the change in magnetic moment along the Bain's transformation path. In addition, the influence of the surfaces, which strongly affect the magnetic properties, ${ }^{22-27}$ was neglected because the bulk iron model was employed. It is, therefore, worth investigating the intrinsic change in magnetic properties at iron surfaces along the Bain's transformation path.

In this paper, we performed $a b$ initio spin-densityfunctional theory calculations to study magnetism at the iron (001) surface and its response to epitaxial in-plane strain corresponding to the well-known Bain (bcc-fcc) transformation path. The simulations simultaneously included the possible magnetic orderings of the collinear FM, AFM1, AFMD, and NM, and noncollinear SS states for the study of stable magnetic phases. The characteristic magnetism at the surface was discussed in terms of the spin-polarized bonding structures.

\section{COMPUTATIONAL DETAILS}

\section{A. Simulation method}

$A b$ initio (first-principles) spin-density-functional theory calculations were carried out using the Vienna Ab-initio Simulation Package (VASP) code. ${ }^{28,29}$ The electronic wave functions were expanded in plane-waves up to a cut-off energy of $E_{\text {cut }}=360 \mathrm{eV}$. The electron-ion interaction was described by projector-augmented-wave (PAW) potentials ${ }^{30,31}$ that explicitly included the $3 d$ and $4 s$ electrons in the valence states. Since the PAW method realizes not only the computational efficiency of the pseudopotential method but also the accuracy of all-electron scheme that avoids the problems concerning to the linearization of the core-valence exchange interaction, the PAW method provides a correct description of magnetism at transition-metal surfaces. ${ }^{32}$ To evaluate the exchange-correlation energy, we employed the GGA of the 


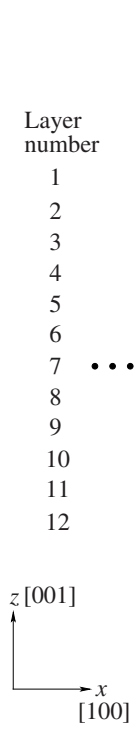

$x$

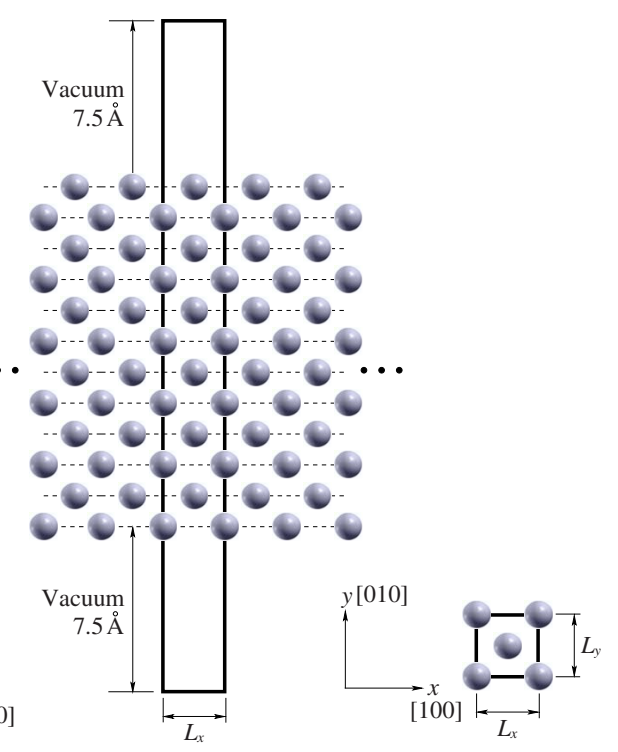

(a) Side View (b) Top View
FIG. 1. (Color online) Simulation model for the Fe (001) surface. The model has a slab geometry with the 12-layered thickness. The solid boxes represent the simulation cell.

Perdew-Burke-Ernzerhof (PBE) functional, ${ }^{21}$ which successfully yielded the structural and magnetic ground state of iron. ${ }^{17,33}$ Within the GGA, the preliminarily calculated lattice constant $a_{0}=2.831 \AA$, magnetic moment $M=2.20 \mu_{B}$, and elastic constants $C_{11}=247 \mathrm{GPa}, C_{12}=143 \mathrm{GPa}$, and $C_{44}$ $=105 \mathrm{GPa}$ for the FM bcc iron bulk were in good agreement with the experimental values ${ }^{34,35}$ of $a_{0}=2.867 \AA$, $M=2.22 \mu_{B}$, and $C_{11}=243 \mathrm{GPa}, C_{12}=139 \mathrm{GPa}$. and $C_{44}=122 \mathrm{GPa}$, respectively.

\section{B. Simulation models and procedure}

The magnetism of the (001) iron surface was studied using a 12-layered slab model, which had sufficient thickness so that undesirable interactions from other surfaces could be neglected. ${ }^{22}$ Figure 1 shows the simulation cell of the 12 atomic-layered Fe film with the (001) surfaces. A threedimensional periodic boundary condition was applied to the simulation cell. The lateral ( $x$ and $y$ ) cell sizes were initially set to the theoretical lattice constant of $\mathrm{Fe}$ bulk, $a_{0}$ $=2.835 \AA$. A vacuum region of $l_{v}=15.0 \AA$ thickness was introduced in the $z$ direction so that interaction among neighboring films due to the periodic boundary condition was negligible. Thus, the initial cell dimensions in the $x, y$ and $z$ directions were $L_{x}=a_{0}, L_{y}=a_{0}$ and $L_{z}=5.5 a_{0}+l_{v}$, respectively. The bulk model of bcc iron with the initial lattice constant of $a_{0} \times a_{0} \times a_{0}$ was also simulated for comparison. The Brillouin zone (BZ) integration was carried out with $14 \times 14 \times 2$ and $14 \times 14 \times 14 k$-point meshes generated by the Monkhorst-Pack scheme ${ }^{36}$ for the film and bulk models, respectively.

To obtain a relaxed surface structure, the atomic positions were fully relaxed using the conjugate gradient $(\mathrm{CG})$ method until all the Hellmann-Feynman forces were less than 1.0 $\times 10^{-3} \mathrm{eV} / \AA$.
To investigate the effects of in-plane epitaxial strain, $\varepsilon$, we applied a small incremental strain equally in both the $x$ and $y$ directions, $\Delta \varepsilon=\Delta \varepsilon_{x x}=\Delta \varepsilon_{y y}$, stepwise to the film and bulk models. This deformation was called the epitaxial Bain's (bcc-fcc) transformation path. The structural relaxation described above was performed at each strain. Because the lattice of the film freely relaxed along the $z$ direction due to the free surface, the stress condition was, therefore, $\sigma_{x x}$ $=\sigma_{y y} \neq 0, \sigma_{z z}=0\left(\sigma_{x x}, \sigma_{y y}, \sigma_{z z}\right.$ : normal stresses $)$. For the fair comparison, the lattice parameter in the $z$ direction of the bulk model was also relaxed so that the stress component of $\sigma_{z z}$ was equal to zero.

To study the stability of complex magnetic phases in iron along the Bain transformation path, we considered the following possible magnetic orderings: collinear FM, NM, single-layer (AFM1- $\uparrow \downarrow \uparrow \downarrow \ldots), \quad$ and double-layer (AFMD- $\uparrow \uparrow \downarrow \downarrow \ldots)^{9,14}$ antiferromagnetic, and noncollinear SS states. For the correct description of the ground state in the noncollinear spin-spiral state, ${ }^{10,12}$ we employed a full vector-field description of magnetization. ${ }^{37}$ In addition, the incommensurate solutions of the SS state were correctly described by applying the generalized Bloch conditions. ${ }^{12,38}$

\section{RESULTS AND DISCUSSION}

\section{A. Magnetism at unstrained surface}

Under the stain-free condition, it is well known that the FM state is the most favorable in the bcc iron because it has the lowest total energy. The calculated total energy difference per atom between the stable FM and other phases is $\Delta E_{\mathrm{AFM} 1-\mathrm{FM}}^{\text {bulk }}=0.407 \mathrm{eV} /$ atom,$\Delta E_{\mathrm{AFMD}-\mathrm{FM}}^{\text {bulk }}=0.175 \mathrm{eV} /$ atom and $\Delta E_{\mathrm{NM}-\mathrm{FM}}^{\mathrm{bulk}}=0.451 \mathrm{eV} /$ atom, and $\Delta E_{\mathrm{AFM} 1-\mathrm{FM}}^{\mathrm{film}}$ $=0.302 \mathrm{eV} /$ atom, $\quad \Delta E_{\mathrm{AFMD}-\mathrm{FM}}^{\mathrm{film}}=0.147 \mathrm{eV} /$ atom, and $\Delta E_{\mathrm{NM}-\mathrm{FM}}^{\mathrm{film}}=0.471 \mathrm{eV} /$ atom, for the bulk and film, respectively. For the noncollinear SS state, the wave vector of $\mathbf{q}=(0,0,0)$ gives the minimum energy, indicating that the FM state is favorable. The strain-free (001) surface in the FM state is, therefore, the focus of the following discussion.

The surface energy, $E_{S}$, can be calculated from the following equation:

$$
E_{S}=\frac{E_{\mathrm{film}}-E_{\mathrm{bulk}} N}{2 S},
$$

where $E_{\text {film }}, E_{\text {bulk }}, N$, and $S$ denote the total energy of the ferromagnetic iron film that of the bulk, the number of atoms and the surface area of the film model, respectively. The calculated surface energy of the (001) surface in the FM phase was $2.48 \mathrm{~J} / \mathrm{m}^{2}$, which is in excellent agreement with $2.47 \mathrm{~J} / \mathrm{m}^{2}$ obtained from a preceding DFT calculation. ${ }^{22}$ The obtained result is also comparable with the available experimental values ${ }^{39,40}$ of 2.41 and $2.55 \mathrm{~J} / \mathrm{m}^{2}$ although these values were obtained for the polycrystalline surface. These agreements demonstrate the reliability of our DFT calculations for the surface.

Figure 2 shows the layer-resolved local magnetic moment in the FM iron film with and without structural relaxation. The structural relaxation leads to change in interlayer distance between the $i$ th and $j$ th layers $\Delta_{i j} ; \Delta_{12}=-0.050 \AA$, 


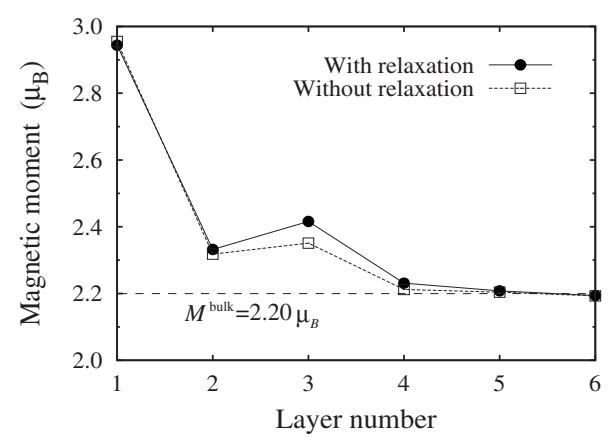

FIG. 2. Layer-resolved local magnetic moment in the FM iron film with and without structural relaxation. The layer number corresponds to that in Fig. 1. Only the top half of the model is shown due to the centrosymmetry in the $z$ direction. The dashed horizontal line indicates the magnitude of the magnetic moment of bulk Fe, $M^{\text {bulk }}=2.20 \mu_{B}$.

$\Delta_{23}=0.036 \AA, \quad \Delta_{34}=0.009 \AA$, and $\Delta_{45}=-0.006 \AA$. The change in interlayer distance calculated in this study is in good agreement with preceding theoretical studies ${ }^{22,24}$ and an experimental observation by a low-energy electron diffraction (LEED). ${ }^{41}$ The local magnetic moments were calculated by projecting the wave functions onto the spherical harmonics within the spheres around each atom, with radii equal to the Wigner-Seitz radii. The figure clearly reveals the enhancement of magnetic moment with respect to that of the bulk in the vicinity of the relaxed surface. The surface atom (layer 1) had the highest magnetic moment of $2.94 \mu_{B}$, which was in good agreement with the value from earlier theoretical studies of the (001) surface 22,42 of $2.95 \mu_{B}$ and $2.98 \mu_{B}$. No remarkable difference could be found in magnetic moments among the unrelaxed and relaxed surface structures, suggesting that the surface relaxation contributed little to the enhancement of magnetic moment.

Figure 3 shows the majority-spin and minority-spin density distributions on the (110) plane in both FM bulk and film. In the bulk, the majority-spin density distribution was

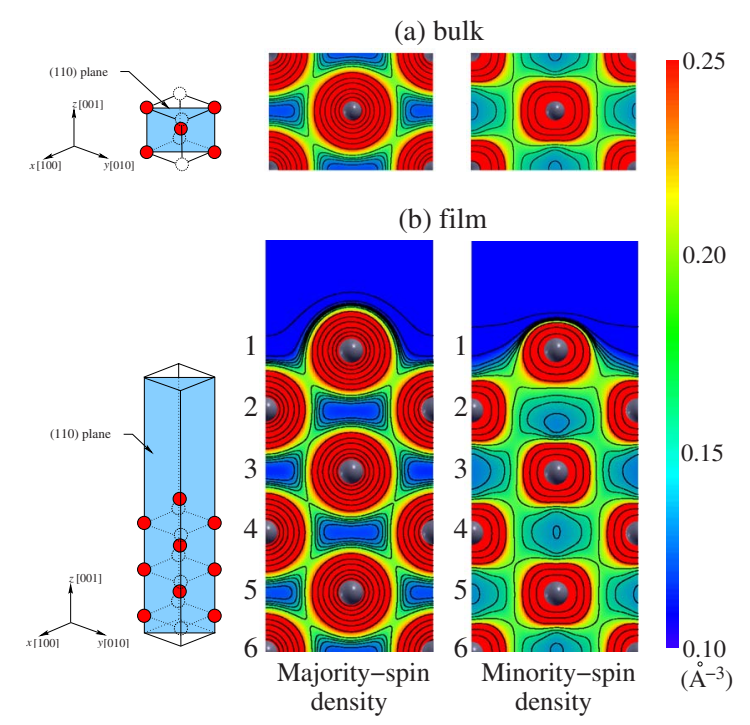

FIG. 3. (Color online) Majority-spin and minority-spin density distributions on the (110) plane in (a) bulk and (b) film.

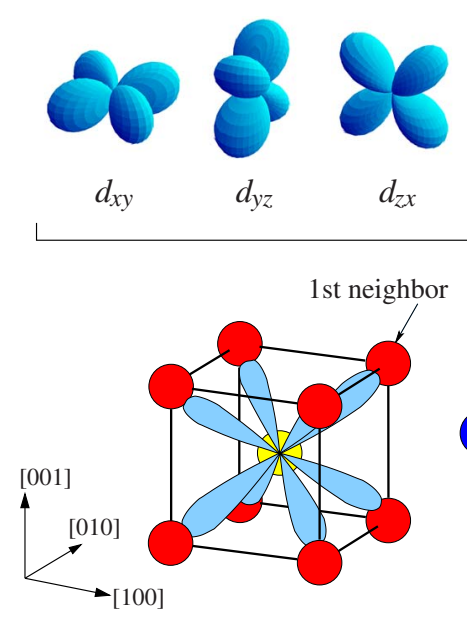

(a) Hybridized $t_{2 g}$ orbit
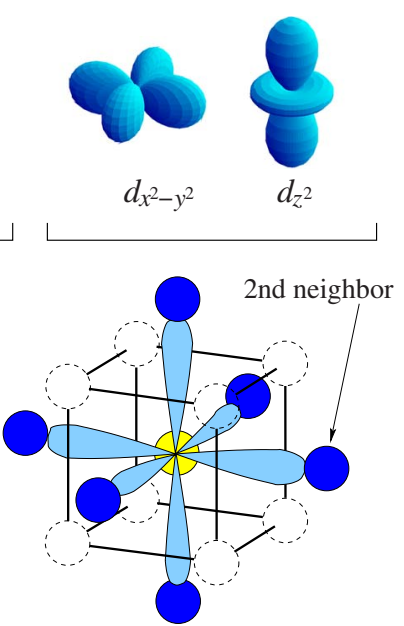

(b) $e_{g}$ orbit
FIG. 4. (Color online) Schematic illustration of (a) hybridized $t_{2 g}\left(d_{x y}, d_{y z}\right.$, and $\left.d_{z x}\right)$ and (b) $e_{g}\left(d_{x^{2}-y^{2}}\right.$ and $\left.d_{z^{2}}\right)$ orbitals in the bcc iron.

almost spherical (isotropic), while a clear anisotropy was found for the minority spin. The distribution of the minority spin was mostly directed toward the nearest neighbors. The atom at the (001) surface of the film (layer 1), on the other hand, lost the directionality in the minority spin toward the surface normal due to the absence of nearest neighbors.

According to crystal-field theory, the five $d$ orbitals, $d_{x y}$, $d_{y z}, d_{z x}, d_{x^{2}-y^{2}}$, and $d_{z^{2}}$, in the bcc crystal reduce to the hybridized $t_{2 g}\left(d_{x y}, d_{y z}\right.$, and $\left.d_{z x}\right)$ and $e_{g}\left(d_{x^{2}-y^{2}}\right.$ and $\left.d_{z^{2}}\right)$ states. ${ }^{43,44}$ The $t_{2 g}$ and $e_{g}$ orbitals are depicted in Fig. 4.

The $t_{2 g}$ electrons distributed toward the eight nearest neighbors in the bcc lattice by forming $d d \sigma$ bonds, ${ }^{45}$ while the $e_{g}$ electrons were directed toward the second-nearest neighbors. From the standpoint of the charge distribution of $t_{2 g}$ and $e_{g}$, the minority-spin density distribution along the nearest-neighbor direction should arise mostly from the $t_{2 g}$ state.

Figure 5 plots the spin-polarized local electronic density of states (DOS) for the total $d$ and $t_{2 g}-e_{g}$ decomposed states in the bulk and at the (001) surface (the atom in layer 1 of the film). The local DOS was calculated by projecting the wave functions onto the spherical harmonics within the spheres around each atom. For the iron bulk, the majority-spin $t_{2 g}$ and $e_{g}$ states were almost fully occupied although there was slight $t_{2 g}$ state above the Fermi level, $E_{\mathrm{F}}$. This suggests that both the $t_{2 g}$ and $e_{g}$ states were almost equitably occupied, which led to no specific directionality in the majority-spin density distribution in Fig. 3(a). On the other hand, the $t_{2 g}$ state was highly occupied in the minority-spin DOS while most of the $e_{g}$ states were located above the Fermi level. This is consistent with the directional minority-spin density distribution toward the nearest neighbors. In the DOS of the (001) surface, a remarkable difference from the bulk was found in the $t_{2 g}$ state: The $t_{2 g}$ states in the majority spin were localized around the lower energy level of $-3.0 \mathrm{eV}$, which resulted in a fully occupied $d$ majority-spin state. On the other hand, the $t_{2 g}$ bands in the minority spin were localized around an energy level slightly higher than $E_{\mathrm{F}}$. Thus, the $t_{2 g}$ state had a 
(a) bulk

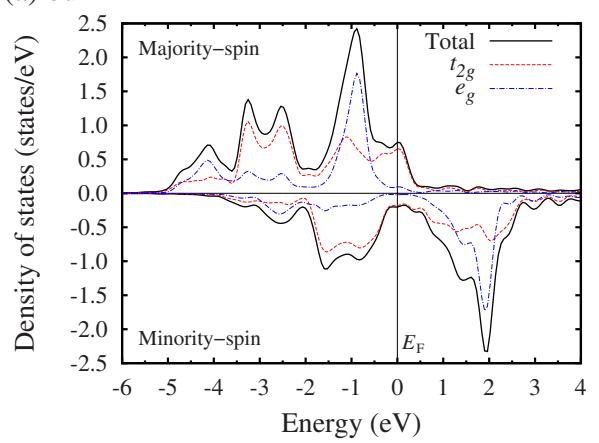

(b) (001) surface

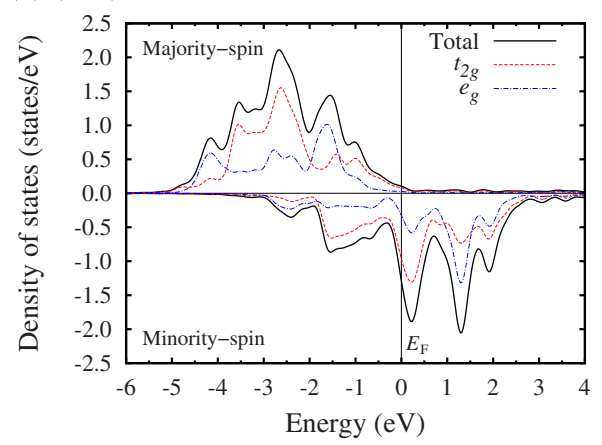

FIG. 5. (Color online) Total $d$ and $t_{2 g}-e_{g}$ decomposed electronic local DOS (a) in the Fe bulk and (b) at the (001) surface. $E_{\mathrm{F}}$ denotes the Fermi level.

reduced bandwidth (the energy-level range that a state occupies), compared to that of the bulk. On the other hand, no difference was eminent in the $e_{g}$ state. It is well known that the bandwidth is reduced as the coordination number decreases. There are four fewer nearest neighbors at the surface than in the bulk (4 vs. 8), while there is only one fewer (6 $\rightarrow 5$ ) second-nearest neighbor. Having 50\% fewer nearest neighbors at the surface results in a significant decrease in the bandwidth of the corresponding $t_{2 g}$ state, while the slight change in the $e_{g}$ DOS arises from the more limited loss of second-nearest neighbors.

As a consequence of the energy-level shift of the $t_{2 g}$ states across the Fermi level between the bulk and the surface, the number of majority-spin and minority-spin electrons changes. Figure 6 shows the number of majority-spin and minority-spin electrons, $N_{\text {majority }}$ and $N_{\text {minority, }}$ respectively, for the $t_{2 g}$ and $e_{g}$ states in the bulk and at the surface. Comparing the (001) surface with the bulk, a significant increase (decrease) in the number of electrons was found in the majority-spin (minority-spin) $t_{2 g}$ states. This indicates that electron rearrangement from minority-spin $t_{2 g}$ to majorityspin $t_{2 g}$ states occurs at the surface layer due to the lower number of nearest neighbors. This results in enhancement of the magnetic moment at the (001) surface, because the magnetic moment is defined as the difference between the number of majority-spin and minority-spin electrons, $M=N_{\text {majority }}-N_{\text {minority }}$.

\section{B. Effect of in-plane strain (epitaxial Bain's path)}

Figure 7(a) shows the total energy per atom of the Fe bulk in the collinear FM, AFM1, AFMD, NM, and noncollinear

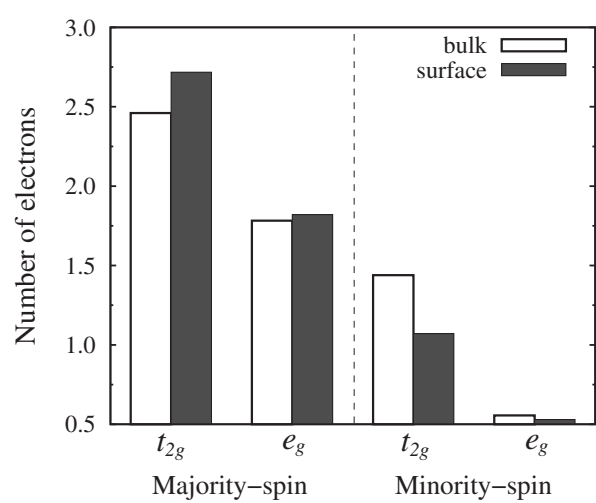

FIG. 6. Number of majority-spin and minority-spin electrons for the $t_{2 g}$ and $e_{g}$ states in the bulk and at the (001) surface.

$\mathrm{SS}$ phases as a function of in-plane epitaxial strain, $\varepsilon$. Since the total energy of the SS phase depends on the wave vector, $\mathbf{q}$, we took the minimum energy at each applied strain along the $\Gamma-X-W$ path $^{12}$ shown in Fig. 7(b). Note that, since the spin-spiral wave vector of $\mathbf{q}=(2 \pi / a) \times(0,0,0)$, which corresponds to the collinear FM state, gives the minimum energy along the path at the strain region of $\varepsilon \geq-0.09$, the SS plots are presented only at $\varepsilon \leq-0.10$. The FM phase is favorable under tension, while the magnetic phase transition from the FM to AFMD occurs at a strain of $\varepsilon=-0.09$ under compression. This result is consistent with the previous studies $^{12,14}$ of the energetics of magnetic phases along the Bain's transformation path. Note that the AFM1 phase is
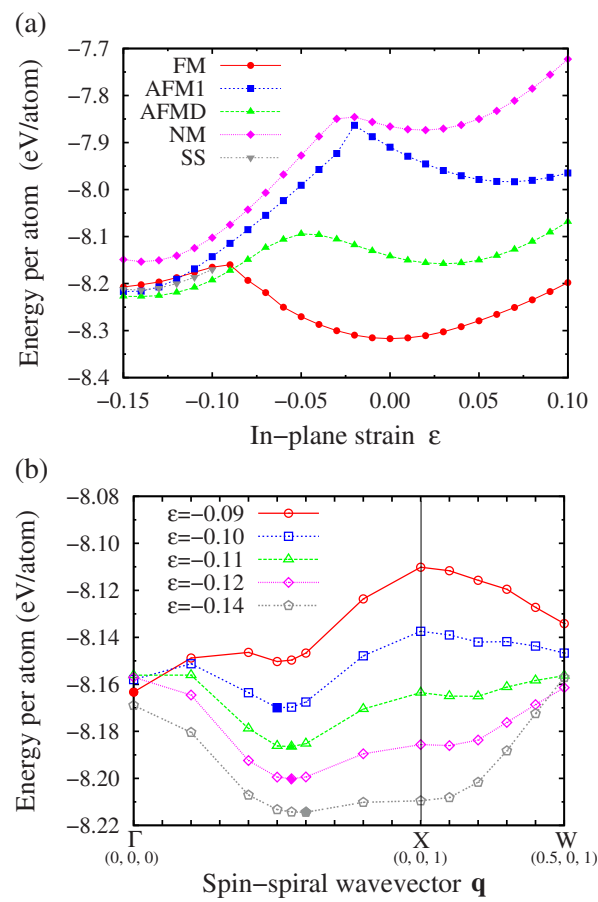

FIG. 7. (Color online) (a) Total energy per atom as a function of in-plane strain, $\varepsilon$, of bulk Fe in the collinear FM, AFM1, AFMD, $\mathrm{NM}$, and noncollinear SS phases. (b) Total energy per atom as a function of spin-spiral wave vector, $\mathbf{q}$, of the Fe bulk at each inplane strain. The SS wave vector is taken along the $\Gamma-X-W$ path. The filled symbols indicate the minimum at each applied strain. 
(a)

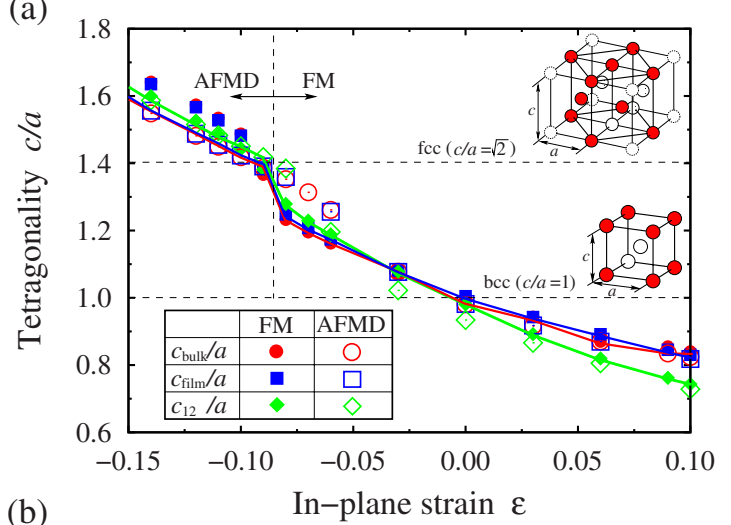

(b)

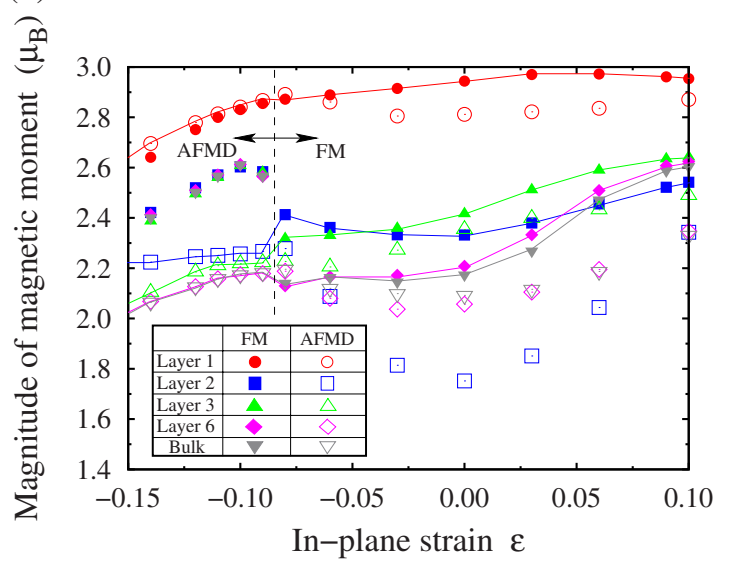

FIG. 8. (Color online) (a) Tetragonality of lattice, $c / a$, as a function of in-plane strain, $\varepsilon$, of the iron bulk and film. $c_{12}$ denotes the $z$-lattice parameter, which corresponds to the interlayer distance between layers 1 and 2 of the film, $d_{12}=c_{12} / 2$. (b) Magnitude of magnetic moment as a function of in-plane strain, $\varepsilon$, of the iron bulk and film. The stable phase structure at each strain is shown by the solid lines; the FM phase for $\varepsilon \geq-0.08$ and the AFMD phase for $\varepsilon \leq-0.09$. See also Fig. 7(a).

energetically unstable with respect to the AFMD phase at all the strain, because the AFM1 can be stabilized only at much lower atomic volume. ${ }^{14}$

Figure 8(a) shows the tetragonality of the lattice, $c / a$, as a function of in-plane strain, $\varepsilon$, of the iron bulk and film, where $a$ and $c$ denote the lattice parameters in the lateral $x$ and $y$ directions and in the $z$ direction, respectively. A local structural change at the surface was also examined by introducing the lattice parameter of the surface layer, $c_{12}=2 d_{12}$, where $d_{12}$ is the interlayer distance between layers 1 and 2 . The tetragonality was initially $c / a=1$ in the unstrained condition, indicating the bcc structure. The $c / a$ increased nearly linearly as compressive strain was applied. At a strain of $\varepsilon$ $=-0.09$ where the FM-AFMD magnetic phase transition occurred, an abrupt increase in $c / a$ over $\sqrt{2}$ was observed. This indicates that the change in crystalline structure from bcc to fcc is related to the phase transition because both phases have different volume at the same strain. The structure of the AMFD phase is much closer to the fcc than that of the FM phase before and onset of the phase transition [see the open symbols in Fig. 8(a)]. Note that this change in $c / a$ was essentially the same for the bulk and film. The change in $c_{12} / a$ (a) (110) cross-section
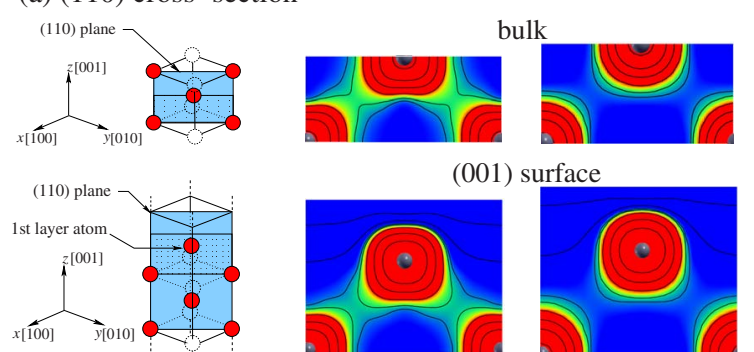

(001) surface

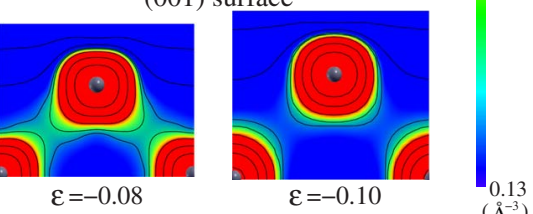

(b) (100) cross-section

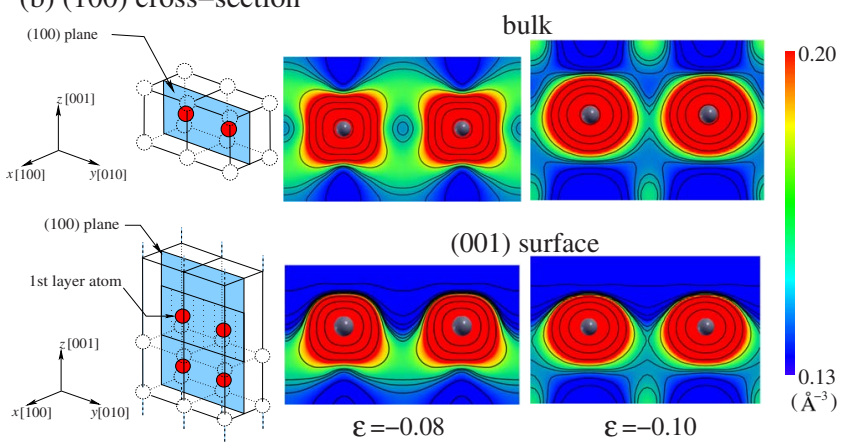

FIG. 9. (Color online) Change in the minority-spin density distribution during the FM bcc $(\varepsilon=-0.08)-$ AFMD fcc $(\varepsilon=-0.10)$ transition in the bulk and at the (001) surface. Top and bottom panels represent the (110) and (100) planes for the nearest neighbors and second-nearest neighbors, respectively.

indicates that the structure at the surface also changed to fcc at the critical strain.

Figure 8(b) shows the magnitude of the magnetic moment as a function of in-plane strain, $\varepsilon$, in the iron bulk and film. The stable FM and AFMD phase structures were taken for $\varepsilon \geq-0.08$ and $\varepsilon \leq-0.09$, respectively. The applied tensile inplane strain tended to enhance the magnetic moments while they were suppressed by compression in both the bulk and film. Because of the magnetic and structural phase transition from FM-bcc to AFMD-fcc, the magnetic moment for the bulk and the inner layers of the film exhibited a discontinuous change at the critical strain of $\varepsilon=-0.09$. On the contrary, the magnetic moment at the surface layer was rather insensitive to this transition, in spite of the abrupt change in crystalline structure.

Figure 9 depicts the change in minority-spin density distribution during the FM bcc $(\varepsilon=-0.08)-$ AFMD fcc $(\varepsilon=$ $-0.10)$ transition, both in the bulk and at the (001) surface. The charge density along the nearest-neighbor directions in the (110) plane became sparse in both the bulk and at the surface, indicating that the $d d \sigma$ bond among the nearest neighbors was considerably weakened during the transition. On the contrary, the charge density increased along the second-nearest-neighbor direction in the (100) plane. This change in the electron distribution suggests that the occupied electronic state of $t_{2 g}$ in the minority spin increases while that of $e_{g}$ decreased.

Figure 10 shows the change in the number of majorityspin and minority-spin electrons in the $t_{2 g}$ and $e_{g}$ states as a function of in-plane strain, $\varepsilon$, in the bulk and at the (001) 
(a) bulk

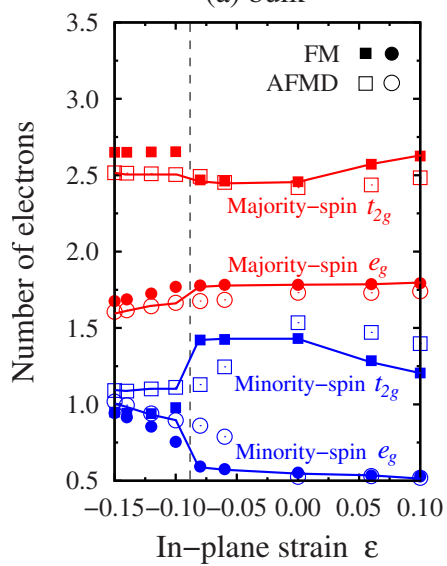

(b) (001) surface

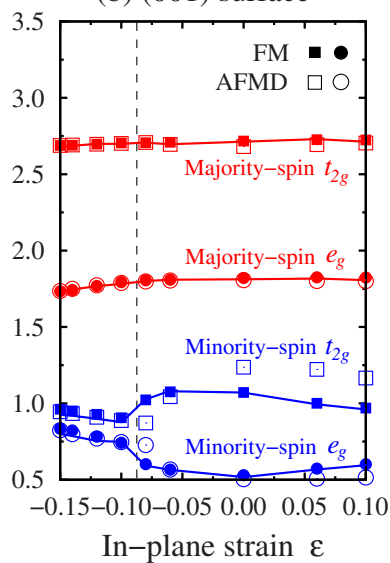

FIG. 10. (Color online) Change in the number of majority-spin (red or dark gray) and minority-spin (blue or black) electrons in the $t_{2 g}$ and $e_{g}$ states as a function of in-plane strain, $\varepsilon$, (a) in the bulk and (b) at the (001) surface. The vertical dashed lines indicate the FM bcc-AFMD fcc transition. The full and open symbols indicate the FM and AFMD phases, respectively. The stable phase structure at each strain is shown by the solid lines; the FM phase for $\varepsilon \geq$ -0.08 and the AFMD phase for $\varepsilon \leq-0.09$. See also Fig. 7(a).

surface. In the bulk, the number of minority-spin and majority-spin electrons discontinuously changed at the transition strain of $\varepsilon=-0.09$, which led to an abrupt change in the magnetic moment of the bulk. On the other hand, the number of majority-spin electrons was almost unchanged and electron rearrangement was limited to within the minority-spin state at the surface. Figure 11 plots the change in the local electronic density of states (DOS) of the $t_{2 g}$ and $e_{g}$ states between the in-plane strains of $\varepsilon=-0.08(\mathrm{FM})$ and $\varepsilon=-0.10$ (AFMD) in the bulk and at the (001) surface. Unoccupied $t_{2 g}$ and $e_{g}$ states existed for both the majority and minority spins in the bulk during the FM-AFMD transition while the majority-spin state remained fully occupied at the (001) surface because of its low coordination number. The electron rearrangement was, therefore, limited to within the minority-spin state, which limited the change in the magnetic moments at the surface.

\section{CONCLUSION}

$A b$ initio (first-principles) spin-density-functional theory calculations within the GGA were carried out to investigate magnetism at the iron (001) surface and its response to the epitaxial in-plane strain that corresponds to the well-known Bain transformation path. The collinear FM, AFM1, AFMD, $\mathrm{NM}$, and noncollinear SS states were included in the calculations along the Bain's path.

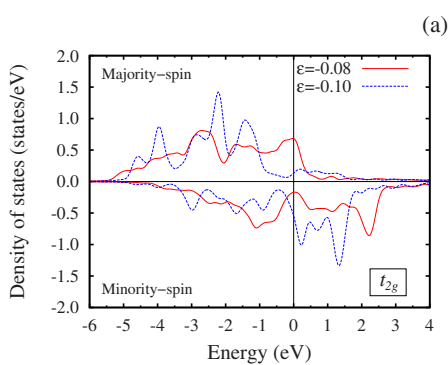

(a) bulk

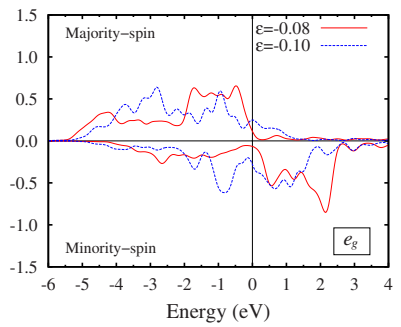

(b) (001) surface
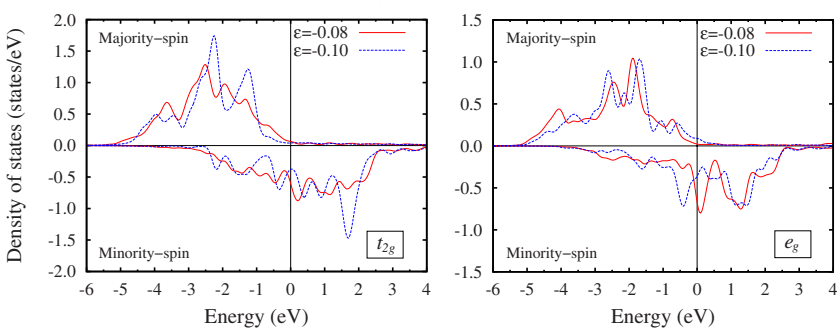

FIG. 11. (Color online) Change in local electronic DOS for the $t_{2 g}$ and $e_{g}$ states between the in-plane strains of $\varepsilon=-0.08(\mathrm{FM})$ and $\varepsilon=-0.10$ (AFMD) (a) in the bulk and (b) at the (001) surface.

At the strain-free (001) surface of FM film, the magnetic moment was enhanced to be $2.94 \mu_{B}$ over that of the bulk, $2.20 \mu_{B}$. This was mainly because of electron rearrangement from the minority-spin $t_{2 g}$ to the majority-spin $t_{2 g}$ state at the surface caused by the reduced coordination number of nearest neighbors.

In-plane tensile strain tended to enhance the magnetic moments, maintaining the FM state during tension, while a structural and magnetic phase transition from FM-bcc to AFMD-fcc occurred under a compressive strain of $\varepsilon=-0.09$, which was accompanied by directional bond switching from nearest-to second-nearest neighbor in the minority spin. As a result, the magnetic moment of the inner layers of the film changed discontinuously due to the abrupt structural and magnetic changes. On the other hand, that of the surface layer was insensitive during the transition. This is because electron exchange from the $t_{2 g}$ to $e_{g}$ states during the transition was limited to within the minority-spin state due to the fully occupied majority-spin state.

\section{ACKNOWLEDGMENTS}

This work was supported in part by a Grant-in-Aid for Scientific Research (S) (Grant No. 21226005) and a Grantin-Aid for Young Scientists (B) (Grant No. 21760073) of the Japan Society of the Promotion of Science (JSPS). 
*shimada@cyber.kues.kyoto-u.ac.jp

${ }^{1}$ G. Autès, C. Barreteau, D. Spanjaard, and M. C. Desjonquères, J. Phys.: Condens. Matter 18, 6785 (2006).

${ }^{2}$ M. Zelený, M. Šob, and J. Hafner, Phys. Rev. B 79, 134421 (2009).

${ }^{3}$ M. Zelený, M. Šob, and J. Hafner, Phys. Rev. B 80, 144414 (2009).

${ }^{4}$ G. A. Prinz, Science 282, 1660 (1998).

${ }^{5}$ F. Li, R. M. Metzger, and W. D. Doyle, IEEE Trans. Magn. 33, 3715 (1997).

${ }^{6}$ K. K. Lew and J. M. Redwing, J. Cryst. Growth 254, 14 (2003).

${ }^{7}$ Y. C. Wang, I. C. Leu, and M. H. Hon, J. Cryst. Growth 237, 564 (2002).

${ }^{8}$ D. Qian, X. F. Jin, J. Barthel, M. Klaua, and J. Kirschner, Phys. Rev. Lett. 87, 227204 (2001).

${ }^{9}$ D. Spišák and J. Hafner, Phys. Rev. B 61, 16129 (2000).

${ }^{10}$ K. Knöpfle, L. M. Sandratskii, and J. Kübler, Phys. Rev. B 62, 5564 (2000).

${ }^{11}$ D. Spišák and J. Hafner, Phys. Rev. B 66, 052417 (2002).

${ }^{12}$ M. Marsman and J. Hafner, Phys. Rev. B 66, 224409 (2002).

${ }^{13}$ M. Friák, M. Šob, and V. Vitek, Philos. Mag. 83, 3529 (2003).

${ }^{14}$ M. Friák, M. Šob, and V. Vitek, Phys. Rev. B 63, 052405 (2001).

${ }^{15}$ L. Tsetseris, Phys. Rev. B 72, 012411 (2005).

${ }^{16}$ M. Friák and M. Šob, Phys. Rev. B 77, 174117 (2008).

${ }^{17}$ M. Zelený, D. Legut, and M. Šob, Phys. Rev. B 78, 224105 (2008).

${ }^{18}$ S. V. Okatov, A. R. Kuznetsov, Y. N. Gornostyrev, V. N. Urtsev, and M. I. Katsnelson, Phys. Rev. B 79, 094111 (2009).

${ }^{19}$ H. C. Herper, E. Hoffmann, and P. Entel, Phys. Rev. B 60, 3839 (1999).

${ }^{20}$ J. P. Perdew and Y. Wang, Phys. Rev. B 45, 13244 (1992).

${ }^{21}$ J. P. Perdew, K. Burke, and M. Ernzerhof, Phys. Rev. Lett. 77, 3865 (1996).

${ }^{22}$ P. Błoński and A. Kiejna, Surf. Sci. 601, 123 (2007).

${ }^{23}$ A. Stibor, G. Kresse, A. Eichler, and J. Hafner, Surf. Sci. 507-
510, 99 (2002).

${ }^{24}$ P. Błoński and A. Kiejna, Vacuum 74, 179 (2004).

${ }^{25}$ M. Eder, K. Terakura, and J. Hafner, Phys. Rev. B 64, 115426 (2001).

${ }^{26}$ W. T. Geng, M. Kim, and A. J. Freeman, Phys. Rev. B 63, 245401 (2001).

${ }^{27}$ J. Yu, X. Lin, J. W. J. Chen, and W. Huang, Appl. Surf. Sci. 255, 9032 (2009).

${ }^{28}$ G. Kresse and J. Hafner, Phys. Rev. B 47, 558 (1993).

${ }^{29}$ G. Kresse and J. Furthmüller, Phys. Rev. B 54, 11169 (1996).

${ }^{30}$ P. E. Blöchl, Phys. Rev. B 50, 17953 (1994).

${ }^{31}$ G. Kresse and D. Joubert, Phys. Rev. B 59, 1758 (1999).

${ }^{32}$ G. Kresse, W. Bergermayer, and R. Podloucky, Phys. Rev. B 66, 146401 (2002).

${ }^{33}$ E. G. Moroni, G. Kresse, J. Hafner, and J. Furthmüller, Phys. Rev. B 56, 15629 (1997).

${ }^{34}$ J. A. Rayne and B. S. Chandrasekhar, Phys. Rev. 122, 1714 (1961).

${ }^{35}$ P. Villars and L. D. Calvert, Pearson's Handbook of Crystallographic Data for Intermetallic Phases, 2nd ed. (ASM International, Metals Park, OH, 1991).

${ }^{36}$ H. J. Monkhorst and J. D. Pack, Phys. Rev. B 13, 5188 (1976).

${ }^{37}$ D. Hobbs, G. Kresse, and J. Hafner, Phys. Rev. B 62, 11556 (2000).

${ }^{38}$ L. M. Sandratskii, J. Phys.: Condens. Matter 3, 8565 (1991).

${ }^{39}$ W. R. Tyson and W. A. Miller, Surf. Sci. 62, 267 (1977).

${ }^{40}$ A. R. Miedema, Z. Metallkd. 69, 287 (1978).

${ }^{41}$ Z. Q. Wang, Y. S. Li, F. Jona, and P. M. Marcus, Solid State Commun. 61, 623 (1987).

${ }^{42}$ S. Ohnishi, A. J. Freeman, and M. Weinert, Phys. Rev. B 28, 6741 (1983).

${ }^{43}$ J. B. Goodenough, Phys. Rev. 120, 67 (1960).

${ }^{44}$ Y. Q. Xie, Acta Metall. Mater. 42, 3705 (1994).

${ }^{45}$ T. E. Jones, M. E. Eberhart, and D. P. Clougherty, Phys. Rev. Lett. 100, 017208 (2008). 